\title{
Relationship between Levels of Sesquiterpene Lactones in Chicory and Sensory Evaluation
}

\author{
Angeline M. Peters ${ }^{1}$ and Aart van Amerongen ${ }^{2}$ \\ Agrotechnological Research Institute (ATO-DLO), P.O.Box 17, NL-6700AA Wageningen, The Netherlands
}

\begin{abstract}
Additional ndex words. antibodies, ELISA, quality assessment, bitterness, taste intensity, typical chicory flavor
ABSTRACT. In this pilot study, we investigated the relationship between levels of bitter sesquiterpene lactones and sensory evaluation of chicory (Cichorium intybus L.). The levels of two bitter sesquiterpene lactones-lactucopicrin and lactucinlike sesquiterpene lactones-were measured by ELISA in raw and cooked chicory samples from several cultivars. Data were compared with the results of a sensory evaluation on the flavor attributes bitterness, typical chicory flavor, and total flavor intensity of identical chicory samples. Linear regression analysis demonstrated that the levels of lactucin-like sesquiterpene lactones were significantly related to bitterness $(P=0.006)$ and total flavor intensity $(P=0.03)$ attributes in raw chicory samples. When cooked chicory samples were evaluated, the levels of lactucin-like sesquiterpene lactones were significantly related to bitterness $(P=0.002)$, typical chicory flavor $(P<0.001)$, and total flavor intensity $(P=0.009)$ attributes, while lactucopicrin levels were related to bitterness $(P=0.002)$ only. These results show that the ELISA can be useful to predict flavor attributes in chicory.
\end{abstract}

Chicory is appreciated as a vegetable for its fine, slightly bitter taste, caused by sesquiterpene lactones. The bitter thresholds of the various sesquiterpene lactones present in chicory were determined by Van Beek et al. (1990).

Since consumers have become more critical toward flavor of food products, a fast, reliable test that can predict the bittemess of chicory will be of great importance to breeders and at markets.

Sensory evaluation of chicory revealed a high correlation of the levels of various sesquiterpene lactones with the bitterness perceived by a sensory panel (Dirinck et al., 1985; Mazijk-Bokslag et al., 1991; Price et al., 1990). Depending on the sample preparation method used, the highest correlation coefficients were found for lactucin glucoside ( $r=0.8$; Price et al., 1990) or for the sum of lactucin and lactucin glucoside ( $r=0.83$; Mazijk-Bokslag et al., 1991). However, in these studies, the levels of glucosidic sesquiterpene lactones were not measured directly with high-performance liquid chromatography (HPLC). Samples were treated with enzymes or acidified methanol by which the glucosides were converted to their free forms preceding HPLC analysis (MazijkBokslag et al., 1991; Price et al., 1990).

Recently, antibodies that recognize lactucin-like sesquiterpene lactones, lactucopicrin, and their precursors have been developed (Peters et al., 1996a, 1996b). ELISAs based on these antibodies can be used to assess the amount of sesquiterpene lactones and their precursors in chicory extracts without the need of extraction or conversion of the glucosides by acidified methanol or enzymes (Peters and Van Amerongen, 1997a).

In this pilot study, we wanted to investigate if ELISA-based levels of sesquiterpene lactones could predict the bitter taste of

Received for publication 7 May 1997. Accepted for publication 17 Oct. 1997. We acknowledge the help of $\mathrm{R}$. van der Vuurst and $\mathrm{C}$. Wilkinson (ATO-DLO, Wageningen, The Netherlands) with statistical analysis and advice during preparation of this manuscript. We also thank $\mathrm{A}$. Ton (PGF, The Hague, The Netherlands) for providing chicory samples and Ir. C.M. Kolb (Oliemans Punter \& Partners, Utrecht, The Netherlands) for sensory analysis. We are grateful to $\mathrm{J}$. Kroeze (Dept. of Marketing and Marketing Research, Wageningen Agricultural University) for valuable discussions and advice. The cost of publishing this paper was defrayed in part by the payment of page charges. Under postal regulations, this paper therefore must be hereby marked advertisement solely to indicate this fact. 'Current address: Department of the Science of Food of Animal Origin, Section Food Chemistry, Faculty of Veterinary Medicine, University Utrecht, P.O. Box 80175, NL-3508 TD Utrecht, The Netherlands.

${ }^{2}$ To whom reprint requests should be addressed. chicory accurately. Data from a sensory evaluation performed at the laboratory of Oliemans, Punter and Partners (Utrecht, The Netherlands) were compared with our ELISA data. The samples were evaluated by a sensory panel on the attributes of bitterness, typical chicory flavor, and total flavor intensity perceived while eating. As chicory is normally consumed raw as well as cooked, both preparation methods were analyzed. Data from the sensory and ELISA analysis were studied by linear regression analysis, which is better fitted to investigate the relationships between sensory attributes and instrumental analysis than calculation of correlation coefficients only.

\section{Materials and Methods}

Cellulase Onozuka (isolated from Trichoderma viride L., $1 \mathrm{U} / \mathrm{mg}$ ) and Macerozyme R10 (pectolytic enzymes isolated from Rhizopus species, $0.5 \mathrm{U} / \mathrm{mg}$ ) were from E. Merck (Darmstadt, Germany). All other chemicals used were reagent grade or higher quality.

Chicory sample preparation. 'Focus', 'Salsa', and 'Sigma' chicory were cultivated at two locations-Lelystad and Zwaagdijk. Roots were cultivated with normal nitrogen manuring (N0; 75 and $117 \mathrm{~kg} \cdot \mathrm{ha}^{-1}$ for Lelystad and Zwaagdijk, respectively) or with additional manuring $\left(\mathrm{N} 1,75+96\right.$ and $117+141 \mathrm{~kg} \cdot \mathrm{ha}^{-1}$ for Lelystad and Zwaagdijk, respectively). Chicory endives that resulted from forcing these roots were used for the sensory analysis. Forcing the roots from Lelystad was repeated after 2 months. From each cultivar, 40 chicory endives were sliced, mixed, and kept refrigerated until use.

SEnsory analysis. The sensory evaluation was performed at the laboratory of Oliemans, Punter and Partners. A panel of 13 trained individuals tasted portions of a $150 \mathrm{-g}$ sample once. Samples were tested raw and after 5 min of cooking with $10 \mathrm{~mL}$ of water. Cooked samples were separated from the boiling water and served at $60^{\circ} \mathrm{C}$. Six samples were tested by each panelist per session. In the first experiment, 12 raw samples were offered randomized to each panelist in two daily sessions. This was repeated with the cooked samples in two further sessions. In the second experiment, six samples were tested raw and cooked in two daily sessions, respectively. The samples were scored for bitterness, typical chicory flavor, and total flavor intensity attributes on a scale of 0 to 100 .

EusSa. Samples of $150 \mathrm{~g}$ of sliced chicory were pretreated as described by Peters et al. (1997b). After grinding $150 \mathrm{~g}$ from each sample under liquid nitrogen, 5-g portions were weighed accu- 
rately and resuspended in an equal volume (w/v) of water. To each sample, $5 \mathrm{mg}$ of cellulase Onozuka and $5 \mathrm{mg}$ of Macerozyme were added and the preparation was incubated for $2 \mathrm{~h}$ at $40^{\circ} \mathrm{C}$. After incubation, the samples were centrifuged for $5 \mathrm{~min}$ at $16,000 \mathrm{~g}_{\mathrm{n}}$. Supernatant was stored at $-20^{\circ} \mathrm{C}$ until use in ELISA.

The levels of lactucin-like sesquiterpene lactones and lactucopicrin were determined with ELISAs using polyclonal antibodies (no. 455) and monoclonal antibodies (no. 4H10), respectively (Peters et al., 1996a, 1996b).

STATISTICAL ANAL YSIS OF SENSORY AND EliSA DATA. To compensate for the variability in the use of the scale by each panelist, $z$ scores were calculated for sensory data. The ELISA data sets and the mean values of sensory z scores were subjected to analysis of variance (ANOVA). Since the data sets were unbalanced, a subset containing the data of the first forcing and a subset containing the data from location Lelystad were used to determine the least significant differences among cultivars and cultivation methods. To study the usefulness of sesquiterpene lactones levels for the prediction of sensory attributes, linear regression analysis was applied to study the relationships between sensory data and ELISA data. The computer program Genstat 5 (release 3.1) was used for statistical analysis of data (Genstat 5 Committee, Rothamstead, U.K.).

\section{Results}

ELISA AND SENSORY ANALYSIS. Levels of lactucin-like sesquiterpene lactones and lactucopicrin in chicory samples were determined with ELISA (Table 1). The overall mean levels for the lactucin-like sesquiterpene lactones were 150 and $52 \mathrm{mg} \cdot \mathrm{kg}^{-1}$ fresh mass for raw and cooked chicory, respectively. The overall mean levels for lactucopicrin in raw and cooked samples were 62 and 40 $\mathrm{mg} \cdot \mathrm{kg}^{-1}$ fresh mass, respectively. Three flavor attributes-bitterness, particular chicory flavor, and total flavor intensity-were used for sensory profiling of the chicory samples (Table 1).

ANOVA on the ELISA data showed that the levels of lactucinlike sesquiterpene lactones and lactucopicrin in the raw chicory samples were significantly $(P<0.001)$ higher than the levels in the cooked samples for both the subsets containing the data from forcing 1 (subset A) and containing the data from location Lelystad (subset B) (Table 2). In subset $A$, the levels of lactucin-like sesquiterpene lactones in samples grown at location Lelystad were significantly $(P=0.002)$ higher than the levels in samples grown at Zwaagdijk. ANOVA of subset B showed that the levels of this sesquiterpene lactone varied significantly $(P<0.001)$ between samples from the first forcing and samples from the second forcing, 2 months later. In addition, a significant $(P=0.032$ and 0.021 ) effect on the lactucin-like sesquiterpene lactone level was found for the interaction of cultivar with nitrogen manuring for subsets $A$ and $B$, respectively.

For the lactucopicrin levels in subset A, significant effects were found for the interactions of cultivar with preparation $(P=0.003)$, nitrogen manuring ( $P=0.004)$, and location $(P=0.001)$ (Table 2 ). In addition, significant effects were found for the interactions of nitrogen manuring with preparation $(P=0.014)$ and location $(P=$ 0.044 ). In subset $B$, significant effects on the lactucopicrin levels were found for the interactions of cultivar and preparation with manuring ( $P=0.011$ and 0.018 , respectively) and forcing $(P=$ 0.004 and 0.022 , respectively).

ANOVA on the mean $\mathrm{z}$ scores in subset $\mathrm{A}$ of the flavor attributes showed that the scores for bitterness varied significantly between locations of cultivation and nitrogen manuring (Table 2). Samples grown at Lelystad or with normal nitrogen manuring (NO) had significantly ( $P=0.012$ and 0.004 , respectively) higher bitterness scores than samples grown at Zwaagdijk or with additional nitrogen manuring (N1). The mean z scores in subset A for the typical chicory flavor varied significantly between locations ( $P$ $<0.001)$ and manuring levels $(P=0.010)$ (Table 2). Again, samples grown at Lelystad or with manuring level N0 showed higher scores than samples grown at Zwaagdijk or with additional manuring (N1). The mean $\mathrm{z}$ scores in subset $\mathrm{B}$ for total flavor intensity varied significantly $(P=0.010)$ between cultivars (Table 2). 'Focus' had higher z scores than 'Salsa' and 'Sigma'.

Table 1. Mean levels of lactucin-like (Lac) and lactucopicrin (Lcp) sesquiterpene lactones (in $\mathrm{mg}^{-\mathrm{kg}^{-1}}$ fresh mass) and mean score ratings (with SD) of the sensory attributes, bitterness (Bitter), typical chicory flavor (Chic), and total flavor intensity (Intens) in raw and cooked chicory samples from 'Focus', 'Salsa', and 'Sigma'. Cultivars were grown at two locations-Lelystad (Lely) and Zwaagdijk (Zwalag) - with two nitrogen manuring levels (N0 and N1). Forcing of chicory endives grown in Lelystad was repeated with a 2 -month interval (Time).

\begin{tabular}{|c|c|c|c|c|c|c|c|c|c|c|c|c|c|}
\hline \multirow[b]{2}{*}{ Place } & \multirow[b]{2}{*}{ Time } & \multirow[b]{2}{*}{ Cultivar } & \multirow[b]{2}{*}{$\mathrm{N}$} & \multicolumn{5}{|c|}{ Raw chicory } & \multicolumn{5}{|c|}{ Cooked chicory } \\
\hline & & & & $\overline{\mathrm{Lac}}$ & Lcp & Bitter & Chic & $\overline{\text { Intens }}$ & $\overline{\mathrm{Lac}}$ & Lcp & Bitter & Chic & Intens \\
\hline \multirow[t]{6}{*}{ Lely } & 1 & Focus & 0 & 189 & 63 & $56(15)$ & $52(16)$ & $53(18)$ & 69 & 38 & $57(17)$ & $54(16)$ & $54(15)$ \\
\hline & & & 1 & 191 & 57 & $58(13)$ & $49(17)$ & $51(14)$ & 79 & 42 & $55(19)$ & $57(16)$ & $56(15)$ \\
\hline & & Salsa & 0 & 171 & 47 & $58(15)$ & $53(19)$ & $51(16)$ & 76 & 35 & $53(17)$ & $55(16)$ & $55(15)$ \\
\hline & & & 1 & 129 & 23 & $40(13)$ & $42(16)$ & $44(14)$ & 51 & 24 & $48(12)$ & $52(10)$ & $51(12)$ \\
\hline & & Sigma & 0 & 185 & 66 & $59(14)$ & $57(15)$ & $51(16)$ & 79 & 48 & $55(15)$ & $57(13)$ & $53(15)$ \\
\hline & & & 1 & 165 & 51 & $50(19)$ & $47(18)$ & $53(18)$ & 74 & 47 & $55(14)$ & $54(16)$ & $51(10)$ \\
\hline \multirow[t]{6}{*}{ Zwaag } & 1 & Focus & 0 & 130 & 38 & $51(17)$ & $59(18)$ & $53(14)$ & 66 & 26 & $47(18)$ & $49(15)$ & $53(16)$ \\
\hline & & & 1 & 183 & 43 & $54(20)$ & $53(15)$ & $55(17)$ & 56 & 26 & $47(18)$ & $52(16)$ & $50(14)$ \\
\hline & & Salsa & 0 & 143 & 31 & $49(18)$ & $51(16)$ & $50(17)$ & 49 & 29 & $49(16)$ & 45 (19) & $47(17)$ \\
\hline & & & 1 & 108 & 20 & $46(17)$ & $45(13)$ & $51(16)$ & 38 & 21 & $45(15)$ & $40(13)$ & 47 (14) \\
\hline & & Sigma & 0 & 142 & 38 & $52(19)$ & $49(19)$ & $51(11)$ & 71 & 25 & $49(14)$ & $48(14)$ & $53(12)$ \\
\hline & & & 1 & 145 & 31 & $39(17)$ & $47(21)$ & $48(13)$ & 45 & 25 & $41(15)$ & $40(16)$ & $46(14)$ \\
\hline \multirow[t]{6}{*}{ Lely } & 2 & Focus & 0 & 131 & 78 & $53(15)$ & $58(11)$ & $54(11)$ & 54 & 48 & $58(17)$ & $52(13)$ & $58(10)$ \\
\hline & & & 1 & 170 & 76 & $51(14)$ & $51(19)$ & $54(14)$ & 74 & 58 & $62(18)$ & $57(14)$ & $62(14)$ \\
\hline & & Salsa & 0 & 139 & 79 & $56(20)$ & $53(17)$ & $58(14)$ & 64 & 48 & $56(18)$ & $60(12)$ & $52(10)$ \\
\hline & & & 1 & 140 & 63 & $60(18)$ & $57(13)$ & $54(16)$ & 40 & 77 & $50(20)$ & $50(16)$ & $51(11)$ \\
\hline & & Sigma & 0 & 124 & 64 & $55(19)$ & $58(10)$ & $50(8)$ & 54 & 49 & $53(20)$ & $49(17)$ & $49(17)$ \\
\hline & & & 1 & 115 & 64 & $49(23)$ & $50(17)$ & $50(14)$ & 80 & 53 & $52(17)$ & $54(16)$ & $51(12)$ \\
\hline Mean & & & & 150 & 52 & & & & 62 & 40 & & & \\
\hline
\end{tabular}


RELATIONSHIP BETWEEN SENSORY AND ELISA DATA. The relationships between the mean $\mathrm{z}$ scores of the sensory attributes and the ELISA data were studied with linear regression analysis (Table 3). The relationships between levels of lactucin-like sesquiterpene lactones in raw chicory samples and bitterness and with total flavor intensity were significant ( $P=0.006$ and 0.03 , respectively), with estimated standard deviations of 0.404 and 0.234 , respectively. The levels of lactucopicrin in raw chicory samples showed no significant relationships with bitterness, particular chicory flavor, or total flavor intensity. In cooked chicory samples, the levels of lactucin-like sesquiterpene lactones showed significant relationships with bitterness $(P=0.002)$, particular chicory flavor $(P<$ $0.001)$, and total flavor intensity $(P=0.009)$. The standard deviations were $0.299,0.291$, and 0.301 , respectively. The level of lactucopicrin in cooked chicory had a significant relationship with bitterness $(P=0.002$, SD $=0.293)$.

\section{Discussion}

In this pilot study, the levels of two bitter sesquiterpene lactones-lactucin and lactucopicrin-in chicory samples were measured by ELISA. These data were related to the results of a sensory evaluation of flavor attributes bitterness, typical chicory flavor, and total flavor intensity by a trained panel.

The levels of sesquiterpene lactones varied significantly between different cultivation methods such as location, manuring levels, and forcing times (Table 2). Previously, Peters et al. (1997b) investigated the effect of cultivation methods of chicory roots on the levels of sesquiterpene lactones. In that study, an effect of cultivar and cultivation method on the levels of sesquiterpene lactones was also observed.

The levels of sesquiterpene lactones in the raw chicory samples were significantly higher than the levels in the cooked samples. The ratio between the overall mean of lactucin-like levels of raw and cooked samples was 2.4. For lactucopicrin levels, the ratio between the overall mean of raw and cooked samples was 1.3.
When samples were cooked, part of the sesquiterpene lactones remained in the cooking water and were, therefore, not measured by ELISA. This resulted in lower levels of sesquiterpene lactones per gram fresh mass. This loss of sesquiterpene lactones was likely to vary between different cultivars or even cultivation methods. In addition, sesquiterpene lactones are temperature sensitive and will partially degrade during cooking, resulting in lower levels in cooked than raw samples (Leclercq, 1992; Pazola, 1987).

In contrast to the differences between raw and cooked samples as measured by ELISA, no difference was observed for the scores of the sensory attributes. This was not expected since levels of sesquiterpene lactones have been reported to correlate with bitterness (Dirinck et al., 1985; Mazijk-Bokslag et al., 1991; Price et al., 1990). One possible explanation may be that during chewing of raw chicory samples, not all sesquiterpene lactones were available for sensory evaluation, whereas the samples were thoroughly ground preceding ELISA. Cooking the samples may have disrupted the cell walls and increased the amount of sesquiterpene lactones available for sensory evaluation. On the other hand, the amount of sesquiterpene lactones may have decreased because of instability of the sesquiterpene lactones at higher temperatures. Apparently these two effects resulted in no significant difference between the perceived intensities of sensory attributes of the raw and cooked chicory samples.

Another explanation may be that the ratio between the mean levels of lactucin-like sesquiterpenes and lactucopicrin was 2-fold higher in raw samples than cooked samples. This means that the cooked samples contained relatively more lactucopicrin. In addition, the decrease of the absolute lactucopicrin levels in cooked samples were smaller than the decrease of lactucin-like sesquiterpene lactones. Since the bitter threshold of lactucopicrin is lower than that of the other sesquiterpene lactones, the higher relative amount of lactucopicrin and smaller decrease upon cooking may have compensated for the loss of lactucin-like sesquiterpene lactones in respect to sensory evaluations (Beek et al., 1990).

Significant relationships were found between the levels of

Table 2. Probabilities for $F$ values for ANOVA of ELISA readings for lactucin (Lac) and lactucopicrin (Lcp) and of mean $z$ scores for the sensory attributes, bitterness (Bitter), typical chicory flavor (Chicory), and total flavor intensity (Intensity) for the subsets containing data from forcing 1 (subset A) or containing data from Lelystad (subset B). Samples from three cultivars were grown at two locations (Lelystad and Zwaagdijk) with two nitrogen manuring levels ( $\mathrm{N}$ level). Forcing was repeated with a 2-month interval (forcing). Samples were analysed raw or cooked (preparation).

\begin{tabular}{|c|c|c|c|c|c|c|}
\hline \multicolumn{2}{|c|}{ Subset } & \multirow{2}{*}{$\begin{array}{c}\mathrm{Lac} \\
0.012\end{array}$} & \multirow{2}{*}{$\frac{\text { Lcp }}{<0.001}$} & \multirow{2}{*}{$\begin{array}{c}\text { Bitter } \\
0.120\end{array}$} & \multirow{2}{*}{$\frac{\text { Chicory }}{0.007}$} & \multirow{2}{*}{$\frac{\text { Intensity }}{0.142}$} \\
\hline $\bar{A}$ & Cultivar (C) & & & & & \\
\hline & Preparation $(\mathrm{P})$ & $<0.001$ & $<0.001$ & 0.534 & 0.797 & 0.930 \\
\hline & $\mathrm{N}$ level $(\mathrm{N})$ & 0.132 & $<0.001$ & 0.012 & 0.002 & 0.136 \\
\hline & Location (L) & 0.002 & $<0.001$ & 0.004 & 0.002 & 0.171 \\
\hline & $\mathrm{C} \times \mathrm{P}$ & 0.308 & 0.003 & 0.609 & 0.792 & 0.847 \\
\hline & $\mathrm{C} \times \mathrm{N}$ & 0.032 & 0.004 & $0.07 \mathrm{I}$ & 0.113 & 0.623 \\
\hline & $\mathrm{C} \times \mathrm{L}$ & 0.971 & 0.001 & 0.228 & 0.028 & 0.858 \\
\hline & $\mathrm{P} \times \mathrm{N}$ & 0.655 & 0.014 & 0.487 & 0.227 & 0.522 \\
\hline & $\mathrm{P} \times \mathrm{L}$ & 0.257 & 0.132 & 0.381 & 0.002 & 0.095 \\
\hline & $\mathrm{N} \times \mathrm{L}$ & 0.439 & 0.044 & 0.678 & 0.637 & 0.879 \\
\hline \multirow[t]{10}{*}{ B } & $\mathrm{C}$ & 0.032 & $<0.001$ & 0.473 & 0.853 & 0.010 \\
\hline & $\mathrm{P}$ & $<0.001$ & 0.011 & 0.453 & 0.428 & 0.043 \\
\hline & $\mathrm{N}$ & 0.645 & $<0.001$ & 0.121 & 0.101 & 0.392 \\
\hline & Forcing (F) & $<0.001$ & $<0.001$ & 0.146 & 0.270 & 0.345 \\
\hline & $\mathrm{C} \times \mathrm{P}$ & 0.142 & 0.113 & 0.564 & 0.826 & 0.160 \\
\hline & $\mathrm{C} \times \mathrm{N}$ & 0.021 & 0.011 & 0.384 & 0.773 & 0.100 \\
\hline & $\mathrm{C} \times \mathrm{F}$ & 0.224 & 0.004 & 0.150 & 0.373 & 0.063 \\
\hline & $\mathrm{P} \times \mathrm{N}$ & 0.615 & 0.018 & 0.420 & 0.128 & 0.363 \\
\hline & $\mathrm{P} \times \mathrm{F}$ & 0.028 & 0.022 & 0.592 & 0.218 & 0.796 \\
\hline & $\mathrm{N} \times \mathrm{F}$ & 0.045 & 0.087 & 0.233 & 0.379 & 0.167 \\
\hline
\end{tabular}


Table 3. Relationship of the mean levels of lactucin-like (lac) or lactucopicrin (lep) sesquiterpene lactones (in mg $\mathrm{kg}^{-1}$ fresh mass) in raw and cooked chicory samples with the sensory attributes bitterness, typical chicory flavor (chicory), and total flavor intensity (intensity).

\begin{tabular}{|c|c|c|c|c|c|}
\hline Preparation & Sesquiterpene lactone & Sensory attribute & $V^{7}$ & Significance & $\mathrm{SD}^{y}$ \\
\hline \multirow[t]{6}{*}{$\overline{\text { Raw }}$} & \multirow[t]{3}{*}{ lac } & Bitterness & 10.09 & $* \%$ & 0.404 \\
\hline & & Chicory & 1.59 & vs & 0.343 \\
\hline & & Intensity & 5.66 & $*$ & 0.234 \\
\hline & \multirow[t]{3}{*}{ lcp } & Bitterness & 2.26 & NS & 0.483 \\
\hline & & Chicory & 2.42 & NS & 0.335 \\
\hline & & Intensity & 1.25 & NS & 0.263 \\
\hline \multirow[t]{6}{*}{ Cooked } & \multirow[t]{3}{*}{ lac } & Bitterness & 13.23 & $* *$ & 0.299 \\
\hline & & Chicory & 21.33 & $* * * *$ & 0.291 \\
\hline & & Intensity & 8.80 & $* *$ & 0.301 \\
\hline & \multirow[t]{3}{*}{ lcp } & Bitterness & 14.50 & $* *$ & 0.293 \\
\hline & & Chicory & 3.25 & NS & 0.405 \\
\hline & & Intensity & 3.03 & NS & 0.343 \\
\hline
\end{tabular}

${ }^{\mathrm{V}} \mathrm{VR}=$ variance ratios.

$y_{\mathrm{SD}}=$ estimated standard deviation.

k.s, ${ }^{* * *}, * * *$ Nonsignificant or significant at $P=0.05,0.01$, or 0.001 , respectively.

lactucin-like sesquiterpene lactones in raw chicory samples and the sensory attributes bitterness and total flavor intensity. In cooked chicory samples, the levels of lactucin-like sesquiterpene lactones showed a significant relationship with bitterness, particular chicory flavor, and total flavor intensity. The level of lactucopicrin only showed a relationship with bitterness in cooked chicory samples.

Significant relationships between the levels of sesquiterpene lactones and sensory attribute were found in this study. However, the prediction of the sensory attributes based on the levels of sesquiterpene lactones is difficult. Calculation of $50 \%, 85 \%$, and $95 \%$ confidence intervals showed that the accuracy of prediction of the sensory attributes based on ELISA data was low because of the relative high estimated standard deviations of the significant relationships (data not shown). Thus, when chicory is classified in, for example, three bitterness classes, the chance that a sample is classified incorrectly is high.

A possible explanation for the low predictability observed, is that other compounds in chicory may have played a role in overall flavor. For example, soluble sugar content may have influenced the perception of bitter sesquiterpene lactones in the samples, giving rise to variable scores for sensory attributes (Walters, 1996).

Another explanation may involve the way samples were treated. For sensory evaluation, the chicory endives were sliced and $150 \mathrm{-g}$ portions were used for analysis. This method was chosen because it resembles the preparation method used by consumers. However, with this method the risk of taking a heterogenous sample is high. As shown previously, the sesquiterpene lactones are not distributed equally in the chicory endives (Peters and Van Amerongen, 1996c). The central axis contains more of the sesquiterpene lactones than the rest of the chicory endives. Indeed, the statistical analysis revealed a few outliers. These samples have a high level of sesquiterpene lactones as related to the sensory scores. This high amount may be caused by heterogenous composition of the portions of chicory endives slices taken for sensory evaluation and for ELISA.

The results showed that ELISA data correlated significantly with sensory flavor attributes bitterness, typical chicory flavor, and total flavor intensity. However, in this pilot study, the predictability was shown to be relatively low due to suboptimal sample preparation and the small range of sensory and analytical parameters of the cultivars used. Therefore, these aspects will be of importance in the further optimization of the use of the ELISAs for predicting chicory flavor attributes.

\section{Literature Cited}

Beek, T.A. van, P. Maas, B.M. King, E. Leclercq, A.G.J. Voragen, and A de Groot. 1990. Bitter sesquiterpene lactones from chicory roots. J. Agr. Food Chem. 38:1035-1038

Dirinck, P., P. van Poucke, M. van Acker, and N. Schamp. 1985. Objective measurement of bitterness in chicory heads (Cichorium intybus L.), p. 62-68. In: W. Baltes, P.B. Czedik-Eysenberg, H. Declstra, P. Dirinck, W. Ooghe, and W. Pfannhauser (eds.). Stratcgies in food quality assurance: Analytical, industrial and lcgal aspects. vol 3. De Sikkel, Antwerp, Belgium.

Leclercq, E. 1992. Sescuiterpene lactones and inulin from chicory roots: extraction, identification, enzymalic release and sensory analysis. $\mathrm{PhD}$ thesis, Agricultural Univ,, Wageningen, The Netherlands.

Mazijk-Bokslag, D.M. van, A.B. Cramwinckel, M.L. Essers, and P.C.H. Hollman. 1991. The relationship between the bitter taste and a HPLC profile of chicory. Res. Bul. 91.50, DLO-State Institute for Quality Control of Agricultural Products, Wageningen, The Netherlands.

Pazola, Z. 1987. The chemistry of chicory and chicory-product beverages, p. 19-57. In: R.J. Clarke and R. Macrae (eds.). Coffee. vol. 5. Related beverages. Elsevier Applied Science, London.

Peters, A.M., N. Haagsma, K.-H. Gensch, and A. van Amerongen. 1996 a. Production and characterisation of polyclonal antibodies against the bitter sesquiterpene lactones of chicory (Cichorium intybus L.). J. Agr. Food Chem. 44:3611-3615.

Peters, A.M, N. Haagsma, and A. van Amerongen. 1996b. Monoclonal antibodies against the bitter sesquiterpene lactones lactucin and lactucopicrin from chicory (Cichorium intybus L.). Food Agr. Immunol. $8: 147-156$.

Peters, A.M. and A. van Amerongen. 1996c. Sesquiterpene lactones in chicory (Cichorium intybus L.): Distribution in chicons and effect of storage. Food Res. Intl. 29:439-444.

Peters, A.M. and A. van Amerongen. 1997a. A study on the effects of sample pre-treatment on the amount of sesquiterpene lactones found in chicory (Cichorium intybus L.) by Elisa and HPLC. Z. Lebensm. Unters. Forsch. 204:189-193.

Peters, A.M., N. Haagsma, and A. van Amerongen. 1997b. A pilot study on the effects of cultivation conditions of chicory (Cichorium intybus L.) roots on the levels of sesquiterpene lactones in chicons. Z. Lebensm. Unters. Forsch. 205:143-147.

Price, K.R., M.S. DuPont, R. Shepherd, H.W.-S.Chan, and G.R. Fenwick. 1990. Relationship between the chemical and sensory properties of exotic salad crops-Coloured lettuce (Lactuca sativa) and chicory (Cichorium intybus). J. Sci. Food Agr. 53:185-192.

Walters, D.E. 1996. How are bitter and sweet tastes related? Trends Food Sci. Technol. 7:399-403. 